\title{
On polynomially integrable planar outer billiards and curves with symmetry property
}

\author{
A.Glutsyuk ${ }^{* \dagger}$ E.Shustin ${ }^{\S}$
}

June 22, 2018

\begin{abstract}
We show that every polynomially integrable planar outer convex billiard is elliptic. We also prove an extension of this statement to non-convex billiards.
\end{abstract}

\section{Contents}

1 Introduction, main result and plan of the paper 1.1 Introduction and main result . . . . . . . . . . . 2

1.2 Historical remarks . . . . . . . . . . . . . . 5

2 Complexification of the curve $C$ : singularities and inflection points

3 Relative symmetry property and quadraticity 8

3.1 Relative symmetry property . . . . . . . . . . . . . 9

3.2 Asymptotics of intersection points . . . . . . . . . 10

3.3 Intersections with the germs having the same projective Puiseux exponents. Proof of Theorem 3.6 . . . . . . . . . . . 14

${ }^{*}$ CNRS, France (UMR 5669 (UMPA, ENS de Lyon) and UMI 2615 (Lab. J.V.Poncelet)), Lyon, France. E-mail: aglutsyu@ens-lyon.fr

${ }^{\dagger}$ National Research University Higher School of Economics (HSE), Moscow, Russia

${ }^{\ddagger}$ Supported by part by RFBR grants 13-01-00969-a, 16-01-00748, 16-01-00766 and ANR grant ANR-13-JS01-0010.

${ }^{\S}$ School of Math. Sci., Tel Aviv University, Ramat Aviv, Tel Aviv 69978, Israel. E-mail: shustin@post.tau.ac.il

『Supported by the grant 176/15 from the Israeli Science Foundation. 
4 Invariants of singularities and Plücker formulas. Proofs of Theorems 1.6 and 1.5 19

4.1 Invariants of plane curve singularities . . . . . . . . . . . 20

4.2 Proof of Theorem $1.6 \ldots \ldots \ldots \ldots \ldots$

4.3 Proof of Theorem $1.5 \ldots \ldots \ldots \ldots$

4.4 Appendix: a general corollary of Theorems 3.6 and 1.6 . . . 24

5 Acknowledgements

\section{Introduction, main result and plan of the paper}

\subsection{Introduction and main result}

Let $C \subset \mathbb{R}^{2}$ be a smooth closed strictly convex curve. Let $S$ denote the complement of the ambient plane $\mathbb{R}^{2}$ to the closure of the interior of the curve $C$. The (planar) outer billiard is a dynamical system $\mathcal{T}: S \rightarrow S$ defined as follows. Pick a point $A \in S$. There are two tangent rays to $C$ issued from the point $A$. Let $R$ denote the right tangent ray: the other tangent ray is obtained from $R$ by rotation around the point $A$ of angle between zero and $\pi$. Let $P$ denote the tangency point of the ray $R$ with the curve $C$. By definition, the image $\mathcal{T}(A)$ is the point of the ray $R$ that is symmetric to $A$ with respect to the point $P$.

Outer billiards were introduced by J.Moser in [21] as a toy model for planetary motion. A mechanical interpretation of outer billiard as an impact oscillator was given by Ph. Boyland [9]. For a survey on outer billiards see [24, 25, 26]. It is well-known that the outer billiard map preserves the

standard Euclidean area, and hence, it is a symplectomorphism.

The famous Birkhoff Conjecture deals with convex bounded planar billiards having a smooth boundary: the classical (Birkhoff) billiards with the usual reflection law, the angle of incidence equals the angle of reflection. Recall that a caustic of a planar billiard $\Omega$ is a curve $C$ such that each tangent line to $C$ reflects from the boundary of the billiard to a line tangent to C. A billiard is called Birkhoff caustic-integrable, if a neighborhood of its boundary is foliated by closed caustics. The Birkhoff Conjecture states that the only integrable billiard is an ellipse.

The Birkhoff Conjecture motivated an analogous conjecture for the outer billiards. Namely, let $C \subset \mathbb{R}^{2}$ be a smooth closed strictly convex curve, $S \subset \mathbb{R}^{2}$ be the exterior component of its complement. We say that the corresponding outer billiard map $\mathcal{T}: S \rightarrow S$ is integrable, if some neighborhood $U$ of the curve $C$ in $\bar{S}$ admits a smooth $\mathcal{T}$-invariant function $U \rightarrow \mathbb{R}$ (called a 
first integral) that has no critical points on $C$. It is known that every elliptic outer billiard is integrable. S.L.Tabachnikov's conjecture [27, p. 101] states that the converse is also true: if an outer billiard is integrable, then it is an ellipse. We prove this conjecture for polynomial first integrals.

Definition 1.1 A planar outer billiard is polynomially integrable, if there exists a non-constant polynomial $f(x, y)$ that is invariant under the outer billiard mapping.

Remark 1.2 Polynomiality of the integral is a very strong restriction of the general Tabachnikov's Conjecture. At the same time, the condition of just non-constancy of a polynomial integral does not forbid it to have critical points on the billiard boundary, while the definition of general integrability forbids them.

The main result of the paper is the following theorem.

Theorem 1.3 Let a planar outer billiard generated by a $C^{4}$-smooth strictly convex closed curve $C$ be polynomially integrable. Then $C$ is an ellipse.

A particular case of Theorem 1.3 under some non-degeneracy assumptions was proved by S.L.Tabachnikov [27, theorem 1]. These assumptions imply in particular that the complex projective Zariski closure of the curve $C$ is non-singular. See the end of Subsection 1.2 for more details.

We also prove the following more general theorem on non-convex curves generating multivalued outer billiard mappings, see the next definition.

Definition 1.4 A $C^{k}$-smoothly immersed curve $C \subset \mathbb{R}^{2}$ generates a polynomially integrable multivalued outer billiard, if there exists a non-constant polynomial $f(x, y)$ such that for every $P \in C$ and every $A, B \in T_{P} C$ symmetric with respect to the point $P$ one has $f(A)=f(B)$. The latter polynomial is called an integral of the multivalued outer billiard constructed on the curve $C$.

Theorem 1.5 Let $C \subset \mathbb{R}^{2}$ be a $C^{4}$-smoothly immersed image of either an interval, or a circle, that does not lie in a line. Let $C$ generate a polynomially integrable multivalued outer billiard. Then the curve $C$ lies in a conic.

Theorem 1.3 follows from Theorem 1.5 .

For the proof of Theorem 1.5 we consider the complexification $\mathbb{C}^{2}$ of the real plane and the ambient projective plane $\mathbb{C P}^{2} \supset \mathbb{C}^{2}$. Let $\overline{\mathbb{C}}_{\infty}$ denote the 
infinity line:

$$
\overline{\mathbb{C}}_{\infty}=\mathbb{C P}^{2} \backslash \mathbb{C}^{2}
$$

It is well-known that the polynomial integral $f$ is constant on $C$ (cf. [27], see Proposition 2.1 below). This implies that the projective complex Zariski closure $\gamma$ of the curve $C$ is an algebraic curve lying in a level curve of the complex polynomial $f$; say, in the zero level curve $\Gamma=\{f=0\}$, provided that $\left.f\right|_{C} \equiv 0$. It suffices to show that each nonlinear irreducible component $\alpha$ of the curve $\gamma$ is a conic. S.Tabachnikov's arguments [27] together with their generalization due to M.Bialy and A.E.Mironov [6, section 6] imply that all the singular and inflection points (if any) of the curve $\gamma$ lying in $\alpha$ are contained in the infinity line $\overline{\mathbb{C}}_{\infty}$ (Theorem 2.2 in Section 2). The curve $\gamma$ has the so-called relative symmetry property: for every point $t \in$ $\gamma \cap \mathbb{C}^{2}$ the central symmetry of the affine line $T_{t} \gamma$ with respect to the point $t$ permutes the points of its intersection with a bigger complex algebraic curve $\Gamma \supset \gamma$ (follows from definition and analyticity). We study the local branches of the curve $\alpha$ at points of the intersection $\gamma \cap \overline{\mathbb{C}}_{\infty}$. Each local branch is a nonlinear irreducible germ of analytic curve, thus it admits a local holomorphic parametrization by small complex parameter $t$,

$$
t \mapsto\left(t^{q}, c t^{p}(1+o(1))\right), p, q \in \mathbb{N}, p>q \geq 1, c \neq 0
$$

in local affine chart centered at the base point. We show that the relative symmetry property (and even its weaker, local version) implies that each local branch transverse to $\overline{\mathbb{C}}_{\infty}$ is quadratic: $p=2 q$ (Theorem 3.6 and its corollary). This is done in Section 3 via asymptotic analysis of the relative symmetry property. Finally, $\alpha$ has the two following properties: all its singular and inflection points (if any) lie in the infinity line $\overline{\mathbb{C}}_{\infty}$; each its local branch transverse to $\overline{\mathbb{C}}_{\infty}$ is quadratic. This together with the next new algebro-geometric theorem proved in Section 4 will imply Theorem 1.5 .

Theorem 1.6 Let a nonlinear irreducible algebraic curve $\alpha \subset \mathbb{C P}^{2}$ have neither singular, nor inflection points in an affine chart $\mathbb{C}^{2} \subset \mathbb{C P}^{2}$. Let each of its local branches at every point in $\alpha \cap \overline{\mathbb{C}}_{\infty}$ that is transverse to $\overline{\mathbb{C}}_{\infty}$ (if any) be subquadratic: $p \leq 2 q$ in its above parametrization. Then $\alpha$ is a conic.

The proof of Theorem 1.5 will be given in Subsection 4.3.

Remark 1.7 It is well-known that polynomial integrability of a Birkhoff billiard $\Omega$ is equivalent to the existence of a non-trivial first integral of the billiard flow in a neighborhood in $\left.T \mathbb{R}^{2}\right|_{\bar{\Omega}}$ of the zero section of the bundle 
$\left.T \mathbb{R}^{2}\right|_{\partial \Omega}$ that is analytic in the speed. In more detail here analyticity is required to be uniform so that the convergence radius of its Taylor series in the speed be uniformly bounded from below. A direct reformulation of this assertion for the outer billiards would state that the existence of an analytic first integral of an outer billiard in a neighborhood of its boundary implies the existence of a polynomial integral. This is unknown, but this would reduce the Tabachnikov's Conjecture for analytically integrable outer billiards to the polynomial case and hence, together with the present results, would prove it. To our opinion, the analytic Tabachnikov's Conjecture seems to be of a level comparable to that of the general smooth case.

\section{$1.2 \quad$ Historical remarks}

The classical Birkhoff Conjecture was studied by many mathematicians starting from the famous paper by H.Poritsky [22], where he stated it in print and proved it under the additional assumption that the billiard in each closed caustic near the boundary has the same closed caustics, as the initial billiard (see also [1]). Here we will mention just few of most known results. In 1993 M.Bialy [3] proved that if the phase cylinder of the billiard is foliated (almost everywhere) by continuous curves which are invariant under the billiard map, then the boundary curve is a circle (see also [31]). See also Bialy's papers [4, 5] for similar results on billiards in constant curvature and on magnetic billiards respectively. D.V.Treschev's experiences [28, 29, 30] provide a numerical evidence of existence of the so-called locally integrable billiards, where a germ of the second iterate of the billiard map is conjugated to a rigid rotation (in two and higher dimensions). Recently V.Kaloshin and A.Sorrentino have proved a local version of the Birkhoff Conjecture [18]: an integrable deformation of an ellipse is an ellipse (see [2] for the case of ellipses with small eccentricities). Birkhoff Conjecture motivated the so-called Algebraic Birkhoff Conjecture, which deals with the polynomially integrable planar billiards, where the billiard geodesic flow has a first integral that depends polynomially on the speed components and is non-constant on the unit level hypersurface of the norm of the speed. It states that the only polynomially integrable convex billiard with smooth boundary is an ellipse. The study of the Algebraic Birkhoff Conjecture and its appropriate generalization to (not necessarily convex) billiards with piecewise smooth boundary on surfaces of constant curvature was started by S.V.Bolotin in [10, 11, see also a survey in [19, chapter 5, section 3]. It was proved for polynomial integrals of degrees up to 4 in [7]. Its complete proof for billiards on any

surface of constant curvature was recently obtained as a result of the three 
following papers: two joint papers by M.Bialy and A.E.Mironov [6, 8]; a very recent paper [14] (see also its short version [15]) of the first author of the present article. For more detailed surveys on Birkhoff Conjecture and its algebraic version see [6, 14, 18, and references therein.

For outer billiards a particular case of Theorem 1.3 was proved by S.L.Tabachnikov [27, theorem 1] under the following additional assumptions:

(i) the complex Zariski closure of the curve $C$ in $\mathbb{C P}^{2}$ is a non-singular algebraic curve;

(ii) the gradient $\nabla F$ of the polynomial integral $F$ does not vanish identically on $C$.

Tabachnikov introduced a powerful method that allowed him to show that under assumptions (i) and (ii) all the singular and inflection points (if any) of the complex projective Zariski closure of the curve $C$ lie in the infinity line. M.Bialy and A.E.Mironov introduced a modified version of Tabachnikov's method that allowed them to prove the same result in the general case (their private communication, see Theorem 2.2 below and its proof) and to prove a similar statement in the context of the Algebraic Birkhoff Conjecture in constant curvature [6, 8].

Remark 1.8 The second part of the proof of the Algebraic Birkhoff Conjecture given in [14, 15] uses results of [6, 8, and techniques elaborated in the present article and in the previous paper of the first author [13.

\section{Complexification of the curve $C$ : singularities and inflection points}

The following proposition is a version of a result from [27].

Proposition 2.1 Let $C \subset \mathbb{R}^{2}$ be a $C^{1}$-smoothly immersed image of either an interval, or a circle. Let $C$ generate a polynomially integrable multivalued outer billiard with the integral $f(x, y)$. Then one has $\left.f\right|_{C} \equiv$ const.

Proof For every point $P \in C$ the restriction of the polynomial integral $f$ to the affine tangent line $T_{P} C$ is invariant under the central symmetry with respect to the point $P$, by definition. Hense, its derivative at $P$ vanishes, as does the derivative at 0 of an even function. Thus, the polynomial $f$ has zero derivative along a vector tangent to $C$ at $P$. Therefore, $\left.f\right|_{C} \equiv$ const, by connectivity. This proves the proposition. 
Theorem 2.2 Let $C \subset \mathbb{R}^{2}$ be a $C^{1}$-smoothly immersed image of either an interval, or a circle, that does not lie in a line and generates a polynomially integrable multivalued outer billiard. Its complex projective Zariski closure $\gamma \subset \mathbb{C P}^{2}$ is an algebraic curve. Each nonlinear irreducible component of the curve $\gamma$ contains neither singular, nor inflection points of the affine curve $\gamma \cap \mathbb{C}^{2}$

A particular case of Theorem 2.2 under assumptions (i) and (ii) from Subsection 1.2 was proved by S.L.Tabachnikov in [27] (for convex curves $C$, but his argument works without convexity assumption). M. Bialy and A. E. Mironov have extended his proof to the general case by using their ideas from [6, section 6]. This proof of Theorem 2.2 due to Tabachnikov, Bialy, and Mironov is given below.

Proof of Theorem 2.2. Let $f$ be a polynomial integral normalized so that $\left.f\right|_{C} \equiv 0$. Then $\left.f\right|_{\gamma} \equiv 0$ by definition, and this implies that $\gamma$ is an algebraic curve. Let $\alpha \subset \gamma$ be a nonlinear irreducible component of the curve $\gamma$. Let $\Psi$ be an irreducible polynomial vanishing on $\alpha$. Then

$$
f=g(x, y) \Psi^{m}(x, y), m \in \mathbb{N},\left.g\right|_{\alpha} \not \equiv 0 .
$$

Set 1

$$
F(x, y)=f^{\frac{1}{m}}(x, y)=g^{\frac{1}{m}}(x, y) \Psi(x, y) .
$$

The function $F$ is multivalued algebraic, and any two of its holomorphic leaves differ by multiplication by $m$-th root of unity. Its branching locus is contained in the curve $\{g=0\}$.

For every $P \in \alpha \cap \mathbb{C}^{2}$ and for every two points $A, B \in T_{P} \alpha$ symmetric with respect to the point $P$ one has $f(A)=f(B)$ : this equality holds in the real domain with $P \in C$ (by definition) and extends analytically to the complex domain. This implies that for every point $P \in \alpha$ such that $g(P) \neq 0$ and $P$ is not a singular point of the curve $\alpha$ each leaf of the function $F$ holomorphic on a neighborhood of the point $P$ has local symmetry property: for every $A, B \in T_{P} \alpha$ symmetric with respect to the point $P$ and close enough to it one has $F(A)=F(B)$.

Consider the (multivalued) vector field $V=F_{y} \frac{\partial}{\partial x}-F_{x} \frac{\partial}{\partial y}$, which is tangent to the level curves of the function $F$ and does not vanish identically on $\alpha$. The above local symmetry property is equivalent to the statement that the function

$$
U(x, y, \varepsilon)=F\left(x+\varepsilon F_{y}, y-\varepsilon F_{x}\right)
$$

\footnotetext{
${ }^{1}$ The case, when $m=1$, was treated by S.L.Tabachnikov 27. His arguments were extended to arbitrary $m$ by M.Bialy and A.E.Mironov, who introduced the function $F$ (their private communication, which repeats their arguments from [6, section 6])
} 
is even in $\varepsilon$ for all $P=(x, y) \in \alpha \backslash(\operatorname{Sing}(\alpha) \cup\{g=0\})$. Equivalently, its Taylor series in $\varepsilon$ should contain only even powers of the variable $\varepsilon$. Set

$$
H(F)=F_{x x} F_{y}^{2}-2 F_{x y} F_{x} F_{y}+F_{y y} F_{x}^{2} .
$$

One has

$$
\left.H(F)\right|_{\alpha} \equiv \text { const }
$$

locally for each leaf of the function $F$ over $\alpha \backslash(\operatorname{Sing}(\alpha) \cup\{g=0\})$. Indeed, for every $P=(x, y) \in \alpha \backslash(\operatorname{Sing}(\alpha) \cup\{g=0\})$ the Taylor coefficient at $\varepsilon^{3}$ of the function $U(x, y, \varepsilon)$, which should vanish, equals $\frac{d H(F)}{d V}$ up to constant factor, see [27, lemma 2] and [6, section 6]. Thus, the latter derivative vanishes, and hence, $\left.H(F)\right|_{\alpha} \equiv$ const, since $V$ is tangent to $\alpha$.

The restriction $\left.H(\Psi)\right|_{\alpha}$ coincides with the value of the Hessian quadratic form of the function $\Psi$ on its skew gradient. Recall that $H(F)=g^{\frac{3}{m}} H(\Psi)$ on the curve $\alpha=\{\Psi=0\}$ (see also [27, lemma 2]). Therefore, $H(F)$ vanishes over all the singular and inflection points of the affine curve $\alpha \cap \mathbb{C}^{2}$. For every regular point $A \in \alpha \cap \mathbb{C}^{2}$ that is singular for the curve $\gamma$ one has $g(A)=0$, hence, $H(F)(P) \rightarrow 0$, as $P \rightarrow A$ along the curve $\alpha$. This together with (2.2) implies that $\alpha \cap \mathbb{C}^{2}$ should be a straight line, as soon as it contains either a singular, or an inflection point of the curve $\gamma$. Thus, since $\alpha \cap \mathbb{C}^{2}$ is not a line by assumption, it contains neither singular, nor inflection points of the curve $\gamma$. Theorem 2.2 is proved.

\section{$3 \quad$ Relative symmetry property and quadraticity}

Definition 3.1 Recall that a local branch of an analytic curve $\gamma$ at a point $A \in \gamma$ is an irreducible component of its germ at $A$.

In the present section we study nonlinear local branches of the complex projective Zariski closure $\gamma$ of the curve $C$ at points of the intersection $\gamma \cap \overline{\mathbb{C}}_{\infty}$ and show that each branch transverse to $\overline{\mathbb{C}}_{\infty}$ is quadratic (Theorem 3.6 and its corollary, see Subsection 3.1). Theorem 3.6 is stated in a more general context, for an irreducible germ $b$ of analytic curve at a point $A \in \overline{\mathbb{C}}_{\infty}$ satisfying the so-called local relative symmetry property introduced in Subsection 3.1. In the proof of Theorem 3.6 given in Subsection 3.3 we use preparatory results given partly in [13] on the asymptotics of the intersection points of the tangent line $T_{P} b$ with a given irreducible germ $a$ of analytic curve at $A$, as $P \rightarrow A$ (Propositions 3.8, 3.10 and their corollaries in Subsection 3.2). 


\subsection{Relative symmetry property}

Definition 3.2 Let $\mathbb{C}^{2} \subset \mathbb{C P}^{2}$ be a fixed affine chart. Let $\gamma \subset \mathbb{C P}^{2}$ be an irreducible algebraic curve distinct from a line. We say that $\gamma$ has relative symmetry property, if there exists an algebraic curve $\Gamma \subset \mathbb{C P}^{2}$ containing $\gamma$ such that for every $t \in \gamma \cap \mathbb{C}^{2}$ the intersection $T_{t} \gamma \cap \Gamma \cap \mathbb{C}^{2}$ is symmetric with respect to the point $t$ as a subset of the affine complex line $T_{t} \gamma \cap \mathbb{C}^{2}$ : it is invariant under the central symmetry $x \mapsto-x$ in an affine coordinate $x$ on $T_{t} \gamma$ centered at $t$.

We will also deal with the local version of the relative symmetry property. To state it, let us introduce the following definition.

Definition 3.3 Let $L \subset \mathbb{C P}^{2}$ be a line, and let $A \in L$. A $(L, A)$-local multigerm is a finite union of distinct irreducible germs of analytic curves $b_{1}, \ldots, b_{N}$ (called components) at base points $A_{j} \in L$ such that each germ at $A_{j} \neq A$ is different from the line $L$. (A germ at $A$ can be arbitrary, in particular, it may coincide with the germ $(L, A)$.) The $(L, A)$-localization of an algebraic curve in $\mathbb{C P}^{2}$ is the corresponding $(L, A)$-local multigerm formed by all its local branches of the above type.

Definition 3.4 Let $\mathbb{C}^{2} \subset \mathbb{C P}^{2}$ be a given affine chart. Let $A \in \mathbb{C P}^{2}, b \subset$ $\mathbb{C P}^{2}$ be a nonlinear irreducible germ of analytic curve at $A$. The germ $b$ has (local) relative symmetry property, if there exists a $\left(T_{A} b, A\right)$-local multigerm $\Gamma$ containing $b$ such that for every $t \in b \cap \mathbb{C}^{2}$ close enough to $A$ the intersection $T_{t} b \cap \Gamma \cap \mathbb{C}^{2}$ is symmetric with respect to the point $t$.

Consider an irreducible nonlinear germ $b$ of analytic curve in $\mathbb{C P}^{2}$ at a given point $A$. Let us choose affine coordinates $(z, w)$ centered at $A$ so that the tangent line $T_{A} b$ be the $z$-axis. Then one can find a local bijective parametrization of the germ $b$ by a complex parameter $t \in(\mathbb{C}, 0)$ of the type

$$
\begin{gathered}
t \mapsto\left(t^{q}, c_{b} t^{p}(1+o(1))\right), \quad q=q_{b}, p=p_{b} \in \mathbb{N}, 1 \leq q<p, c_{b} \neq 0 ; \\
q=1 \text { if and only if } b \text { is a smooth germ. }
\end{gathered}
$$

In the case, when $b$ is a germ of line, it is parameterized by $t \mapsto(t, 0)$, and we set $q_{b}=1, p_{b}=\infty$.

Definition 3.5 The projective Puiseux exponent [13, p. 250, definition 2.9] of a nonlinear germ $b$ is the ratio

$$
r=r_{b}=\frac{p_{b}}{q_{b}}
$$


The germ $b$ is called quadratic, if $r_{b}=2$, and is called subquadratic, if $r_{b} \leq 2$. By definition, the projective Puiseux exponent of a germ of line equals infinity.

Theorem 3.6 Let a nonlinear irreducible germ $b$ of analytic curve in $\mathbb{C P}^{2}$ at a point $A \in \overline{\mathbb{C}}_{\infty}$ be transverse to $\overline{\mathbb{C}}_{\infty}$ and have local relative symmetry property. Then it is quadratic.

Theorem 3.6 is proved in Subsection 3.3.

Corollary 3.7 Let a nonlinear irreducible germ $b$ of analytic curve in $\mathbb{C P}^{2}$ at a point $A \in \overline{\mathbb{C}}_{\infty}$ be transverse to $\overline{\mathbb{C}}_{\infty}$ and lie in the complex projective Zariski closure $\gamma$ of a $C^{2}$-smoothly immersed real curve generating a polynomially integrable multivalued outer billiard. Then $b$ is quadratic.

Proof Let $C$ be the real curve under question, and let $f$ be a polynomial integral of the corresponding outer billiard. Without loss of generality we consider that $C$ is an immersed image of a connected curve, either an interval, or a circle (passing to a smaller curve) and $\left.f\right|_{\gamma} \equiv 0$, see Proposition 2.1. Let $\gamma_{0}$ denote the irreducible component of the curve $\gamma$ containing the germ $b$. The component $\gamma_{0}$ is nonlinear and has the relative symmetry property with respect to the algebraic curve $\Gamma=\{f=0\} \supset \gamma$ : for every $P \in \gamma \cap \mathbb{C}^{2}$ the restriction of the integral $f$ to the affine tangent line $T_{P} \gamma$ is invariant under the central symmetry with respect to $P$, as in the proof of Theorem 2.2 ,

For every point $u \in b$ close to $A$ the intersection of the line $T_{u} b$ with the algebraic curve $\Gamma$ coincides with the intersection of the line $T_{u} b$ and the $\left(T_{A} b, A\right)$-localization of the curve $\Gamma$. This is implied by definition and the following facts:

- for any neighborhood $U$ of a given line $L$ all the lines sufficiently close to $L$ are contained in $U$;

- the point of intersection $T_{u} b \cap T_{A} b$ tends to $A$, as $u \rightarrow A$.

The local branch $b$ of the curve $\gamma_{0}$ has local relative symmetry property with respect to the $\left(T_{A} b, A\right)$-localization of the curve $\Gamma$, by the above discussion. This together with Theorem 3.6 implies that $b$ is quadratic and proves the corollary.

\subsection{Asymptotics of intersection points}

Proposition 3.8 Let $a, b$ be transverse irreducible germs of holomorphic curves at the origin in $\mathbb{C}^{2}$, let $b$ be nonlinear. Let $(z, w)$ be affine coordinates 
in a neighborhood of the origin in $\mathbb{C}^{2}$ centered at 0 such that $b$ is tangent to the $z$-axis at 0 . Let $t$ be the local parameter of the curve $b$ as in (3.1): $z(t)=$ $t^{q_{b}} ; w(t)=c_{b} t^{p_{b}}(1+o(1))$. Then for every $t$ small enough the intersection $T_{t} b \cap$ a consists of $q_{a}$ points $\xi_{1}, \ldots, \xi_{q_{a}}$ whose coordinates have the following asymptotics, as $t \rightarrow 0$ :

$$
z\left(\xi_{j}\right)=O\left(t^{p_{b}}\right)=o\left(t^{q_{b}}\right)=o(z(t)), w\left(\xi_{j}\right)=\left(1-r_{b}\right) w(t)(1+o(1)) .
$$

(Recall that $q_{a}=1$, if a is a germ of line.)

Proof It suffices to prove just the second asymptotic formula in (3.2). Indeed, one has $z\left(\xi_{j}\right)=O\left(w\left(\xi_{j}\right)\right)$, by transversality. This together with the second formula in (3.2) implies the first one: $z\left(\xi_{j}\right)=O(w(t))=O\left(t^{p_{b}}\right)$.

For every $t$ small enough the tangent line $T_{t} b$ intersects the $z$-axis at a point $P_{t}$ with the coordinate

$$
z\left(P_{t}\right)=\nu z(t)(1+o(1))=\nu t^{q_{b}}(1+o(1)), \nu=\frac{r_{b}-1}{r_{b}},
$$

by [13, Proposition 2.10, p. 250]. Let $Q_{t}$ denote the intersection point of the line $T_{t} b$ with the $w$-axis. One has

$$
w\left(Q_{t}\right)=\frac{\nu}{\nu-1} w(t)(1+o(1))=\left(1-r_{b}\right) w(t)(1+o(1)) .
$$

Indeed, the triangle with the vertices $P_{t}, t,(z(t), 0)$ is "complex-similar" to the triangle $P_{t} Q_{t} O$ ( $O$ is the origin), since their sides opposite to the vertex $P_{t}$ lie in parallel affine complex lines. That is, in the new affine coordinates centered at $P_{t}$ the second triangle is obtained from the first one by multiplication by the complex number $\frac{z\left(P_{t}\right)}{z\left(P_{t}\right)-z(t)}=\frac{\nu}{\nu-1}(1+o(1))$, see (3.3). This implies (3.4). Let now $a$ be an arbitrary irreducible germ of holomorphic curve at the origin that is transverse to $b$. Every family of points $\zeta(t)$ of the intersection $T_{t} b \cap a$ has $w$-coordinate asymptotically equivalent to $w\left(Q_{t}\right)$, by transversality and since the line $T_{t} b=Q_{t} \zeta(t)$ tends to the $z$-axis. In more detail, suppose the contrary: the above $w$-coordinates are not asymptotically equivalent, hence the difference $w(\zeta(t))-w\left(Q_{t}\right)$ is asymptotically no less than $c w(\zeta(t)), c \neq 0$. Then the ratio $\frac{w(\zeta(t))-w\left(Q_{t}\right)}{z(\zeta(t))}$ would not tend to zero, since $z(\zeta(t))=O(w(\zeta(t)))$, as was mentioned above. Hence, the line $Q_{t} \zeta(t)$ would not tend to the $z$-axis, - a contradiction. This together with (3.4) proves the second equality in (3.2). The proposition is proved. 
Corollary 3.9 Let $A \in \overline{\mathbb{C}}_{\infty}$, and let $(b, A) \subset \mathbb{C P}^{2}$ be a nonlinear irreducible germ of analytic curve that is transverse to $\overline{\mathbb{C}}_{\infty}$. Let $(a, A)$ be another irreducible germ transverse to $b$, and let $\xi_{1}, \ldots, \xi_{q_{a}}, \xi_{j}=\xi_{j}(t)$ be the points of intersection $T_{t} b \cap a$. Let $(x, y)$ be affine coordinates in $\mathbb{C}^{2}$ such that the $x$-axis is tangent to $b$ at $A$. Then

$$
x(t)=o\left(x\left(\xi_{j}(t)\right)\right) \quad \text { for all } j, \quad \text { as } t \rightarrow A .
$$

Proof Take the local coordinates $(z, w)=\left(\frac{1}{x}, \frac{y}{x}\right)$ centered at $A$ and apply the first formula in (3.2).

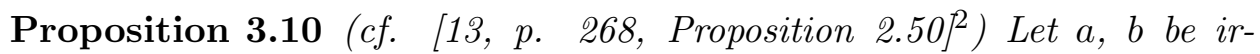
reducible germs of holomorphic curves at the origin in the plane $\mathbb{C}^{2}$ with coordinates $(z, w)$. Let $a$ and $b$ be tangent to the $z$-axis, and let $b$ be nonlinear. Let $c_{a}$ and $c_{b}$ be the corresponding coefficients in (3.1). Then for every $t$ small enough the intersection $T_{t} b \cap a$ consists of $p_{a}$ points $\xi_{1}, \ldots, \xi_{p_{a}}$ (or just one point $\xi_{1}$, if a is the germ of the line $T_{O} b$ ) whose $z$-coordinates have the following asymptotics, as $t \rightarrow 0$.

Case 1): $r_{a}>r_{b}$ (including the linear case, when $r_{a}=\infty$ ). One has

$$
\begin{gathered}
z\left(\xi_{j}\right)=\frac{r_{b}-1}{r_{b}} z(t)(1+o(1))=\frac{r_{b}-1}{r_{b}} t^{q_{b}}(1+o(1)) \text { for } 1 \leq j \leq q_{a}, \\
z(t)=O\left(\left(z\left(\xi_{j}\right)\right)^{\frac{r_{a}-1}{r_{b}-1}}\right)=o\left(z\left(\xi_{j}\right)\right) \text { for } j>q_{a} .
\end{gathered}
$$

(Points satisfying (3.7) exist only when a is nonlinear.)

Case 2): $r_{a}=r_{b}$. One has

$$
z\left(\xi_{j}\right)=\zeta_{j}^{q_{a}} z(t)(1+o(1))=\zeta_{j}^{q_{a}} t^{q_{b}}(1+o(1)),
$$

where $\zeta_{j}$ are the roots of the polynomial

$$
R_{p_{a}, q_{a}, c}(\zeta)=c \zeta^{p_{a}}-r \zeta^{q_{a}}+r-1 ; r=\frac{p_{a}}{q_{a}}>1, c=\frac{c_{a}}{c_{b}} .
$$

(In the case, when $b=a$, one has $c=1$, and the above polynomial has the double root 1 corresponding to the tangency point $t$.)

Case 3): $r_{a}<r_{b}$. One has

$$
z\left(\xi_{j}\right)=O\left((z(t))^{\frac{r_{b}}{r_{a}}}\right)=o(z(t)) .
$$

\footnotetext{
${ }^{2}$ The formulas from loc. cit. provide the inverse expressions, for the coordinate $z(t)$ in terms of $z\left(\xi_{j}(t)\right)$. They are equivalent to the formulas given here.
} 
Proof All the statements of the proposition were proved in loc. cit. except for the statement saying that in Case 1) one has exactly $q_{a}$ intersection points with asymptotics (3.6) and exactly $p_{a}-q_{a}$ intersection points with asymptotics (3.7) (no points satisfying (3.7), if $a$ is linear). Let us prove the latter statement. Thus, we consider that $r_{a}>r_{b}$. The case, when $a$ is linear, was treated in [13, proposition 2.10, p.250], see also formula (3.3) above. Thus, we consider that $a$ is nonlinear. Let $\tau$ denote the value of the local parameter of the curve $a$ at a point of intersection $\xi(t) \in T_{t} b \cap a$. The obvious analytic equality

$$
G(t, \tau)=w(t)+\frac{w^{\prime}(t)}{z^{\prime}(t)}(z(\xi(t))-z(t))-w(\xi(t))=0
$$

has asymptotic form

$$
G(t, \tau)=t^{p_{b}}(1+o(1))+r_{b} t^{p_{b}-q_{b}}\left(\tau^{q_{a}}-t^{q_{b}}\right)(1+o(1))-c \tau^{p_{a}}(1+o(1))=0,
$$

$c=\frac{c_{a}}{c_{b}} \neq 0$, as in [13, p. 269, proof of Proposition 2.50]. The Newton diagram of the germ of analytic function $G(t, \tau)$ is generated by the three monomials: $\left(1-r_{b}\right) t^{p_{b}}, r_{b} t^{p_{b}-q_{b}} \tau^{q_{a}},-c \tau^{p_{a}}$. It consists of two edges: the first one with the vertices $\left(p_{b}, 0\right)$ and $\left(p_{b}-q_{b}, q_{a}\right)$; the second one with the vertices $\left(p_{b}-q_{b}, q_{a}\right)$ and $\left(0, p_{a}\right)$. The latter edges lie on distinct lines. The three above statements on Newton diagram follow from the inequality $r_{a}>r_{b}$, as in loc. cit. The germ of analytic curve $\{G=0\} \subset \mathbb{C}_{(t, \tau)}^{2}$ at the origin is a union of two germs $\eta_{1} \cup \eta_{2}$ corresponding to the edges of the Newton diagram, as in loc. cit. Namely, the monomials $\left(1-r_{b}\right) t^{p_{b}}$ and $r_{b} t^{p_{b}-q_{b}} \tau^{q_{a}}$ generating the first edge are asymptotically opposite (asymptotically "cancel out") along the germ $\eta_{1}$, and all the other Taylor monomials of the function $G$ are of higher order along $\eta_{1}$, as in loc. cit. This implies that for every fixed small $t$ there are exactly $q_{a}$ parameter values $\tau$ for which $(t, \tau) \in \eta_{1}$, and they satisfy asymptotic equality (3.6). Similarly, the monomials $r_{b} t^{p_{b}-q_{b}} \tau^{q_{a}}$ and $-c \tau^{p_{a}}$ are asymptotically opposite along the germ $\eta_{2}$, and for every fixed small $t$ there are exactly $p_{a}-q_{a}$ values $\tau$ such that $(t, \tau) \in \eta_{2}$, and they satisfy asymptotic equality (3.7). Proposition 3.10 is proved.

Corollary 3.11 Let $a, b \subset \mathbb{C P}^{2}$ be irreducible germs of holomorphic curves at a point $A \in \overline{\mathbb{C}}_{\infty}$ that are tangent to each other and transverse to $\overline{\mathbb{C}}_{\infty}$, let $b$ be nonlinear. Let $(x, y)$ be affine coordinates on $\mathbb{C}^{2}$ with the $x$-axis being tangent to $b$ at $A$. Let $\xi_{1}, \ldots, \xi_{p_{a}}, \xi_{j}=\xi_{j}(t)$ be the points of intersection $T_{t} b \cap a$. Their $x$-coordinates have the following asymptotics, as $t \rightarrow A$ : 
Case 1): $r_{a}>r_{b}$. One has

$$
\begin{gathered}
x\left(\xi_{j}\right)=\frac{r_{b}}{r_{b}-1} x(t)(1+o(1)) \text { for } 1 \leq j \leq q_{a}, \\
x\left(\xi_{j}\right)=o(x(t)) \text { for } j>q_{a} .
\end{gathered}
$$

(Points satisfying (3.13) exist only when a is nonlinear.)

Case 2): $r_{a}=r_{b}$. One has

$$
x\left(\xi_{j}\right)=\theta_{j}^{q_{a}} x(t)(1+o(1)),
$$

where $\theta_{j}$ are the roots of the polynomia

$$
\begin{gathered}
Q_{p_{a}, q_{a}, c}(\theta)=\theta^{p_{a}} R_{p_{a}, q_{a}, c}\left(\theta^{-1}\right)=(r-1) \theta^{p_{a}}-r \theta^{p_{a}-q_{a}}+c ; \\
r=\frac{p_{a}}{q_{a}}=\frac{p_{b}}{q_{b}}>1, c=\frac{c_{a}}{c_{b}} .
\end{gathered}
$$

Case 3): $r_{a}<r_{b}$. One has

$$
x(t)=o\left(x\left(\xi_{j}\right)\right) .
$$

The corollary follows from Proposition 3.10 by writing its asymptotics in the local coordinates $(z, w)=\left(\frac{1}{x}, \frac{y}{x}\right)$.

\subsection{Intersections with the germs having the same projective Puiseux exponents. Proof of Theorem 3.6}

Let $A \in \overline{\mathbb{C}}_{\infty}$, and let $b \subset \mathbb{C P}^{2}$ be a nonlinear irreducible germ of analytic curve at $A$ that is transverse to $\overline{\mathbb{C}}_{\infty}$. Let $\Gamma \supset b$ be an arbitrary $\left(T_{A} b, A\right)$-local multigerm containing $b$. Let $(x, y)$ be affine coordinates on $\mathbb{C}^{2}$ such that the $x$-axis is tangent to $b$ at $A$.

Proposition 3.12 Those points of the intersection $T_{t} b \cap \Gamma$ whose $x$-coordinates are asymptotically equivalent to $x(t)$ up to a nonzero multiplicative constant, as $t \rightarrow A$, lie in the intersection of the line $T_{t} b$ with those irreducible germs $a \subset \Gamma$ that are centered at $A$, tangent to $b$ and for which $r_{a} \geq r_{b}$, including the germ b. (Some of these germs a may be linear.) The asymptotics of their $x$-coordinates are given by either (3.12) if $r_{a}>r_{b}$, or 3.14) if $r_{a}=r_{b}$.

\footnotetext{
${ }^{3}$ In the case, when $b=a$, one has $c=1$, and the polynomial $Q_{p_{a}, q_{a}, 1}$ has double root 1 corresponding to the tangency point $t$. It has roots $\theta$ with $\theta^{q_{a}} \neq 1$, if and only if $r=\frac{p_{a}}{q_{a}} \neq 2$.
} 
The proposition follows immediately from Corollaries 3.9 and 3.11 and the fact that the $x$-coordinates of the points of the intersection of the line $T_{t} b$ with those germs $a$ in $\Gamma$ that are not centered at $A$ tend to finite limits, as $t \rightarrow A$, while $x(t) \rightarrow \infty$.

In the sequel we assume that the curve $b$ has relative symmetry property with respect to the multigerm $\Gamma$. First we prove Theorem 3.6 in the next simplest special case in order to underline the main ideas, and then in the general case.

Special case: $\Gamma \backslash b$ is a union of germs centered at $A$ and transverse to $b$. Let us prove quadraticity of the germ $b$. To do this, we consider those intersection points of the tangent line $T_{t} b$ with $\Gamma$, whose $x$-coordinates have asymptotics $\nu x(t)(1+o(1)), \nu \neq 0$, as $t \rightarrow A$. These are exactly the points of the intersection $T_{t} b \cap b$ (Proposition [3.12). Their $x$-coordinates have asymptotics $\theta_{j}^{q} x(t)(1+o(1))$, where $\theta_{1}, \ldots, \theta_{p}$ are the roots of the polynomial $W(\theta)=Q_{p, q, 1}(\theta)=(r-1) \theta^{p}-r \theta^{p-q}+1$; here $p=p_{b}$ and $q=q_{b}$ are the degrees from the parametrization (3.1) of the germ $b$ in the local chart $(z, w)=\left(\frac{1}{x}, \frac{y}{x}\right)$ (Corollary 3.11). The intersection points of the line $T_{t} b$ with the other, transverse germs of the curve $\Gamma$ have $x$-coordinates with bigger asymptotics, by Corollary 3.9. This together with the relative symmetry property implies that the collection of $x$-coordinates of the points of intersection $T_{t} b \cap b$ is invariant under the symmetry with respect to the point $x(t)$. This implies that the collection of powers $\theta_{j}^{q}$ is invariant under the symmetry with respect to 1 . Therefore,

$$
\sum_{j=1}^{p} \theta_{j}^{q}=p,
$$

by symmetry. On the other hand,

$$
\sum_{j=1}^{p} \theta_{j}^{q}=\frac{p}{r-1} .
$$

Indeed, the latter sum is independent of the free term of the polynomial $W$, being expressed via elementary symmetric polynomials of degrees at most $q<p$, which are independent of the free term. Therefore, it equals the sum of the $q$-th powers of nonzero roots of the polynomial $(r-1) \theta^{p}-r \theta^{p-q}$. All the latter $q$-th powers are equal to $\frac{r}{r-1}$, hence their sum equals $\frac{q r}{r-1}=\frac{p}{r-1}$. This proves (3.17). Formulas (3.16) and (3.17) together imply that $p=\frac{p}{r-1}$. Hence, $r=2$.

General case. Let $a_{1}, \ldots, a_{l} \subset \Gamma$ be those irreducible germs that are centered at $A$, tangent to $b$ and have the same projective Puiseux exponent: 
$r_{a_{i}}=r_{b}=r ; b$ is one of them. Here $l$ may be any natural number including 1. Let $q_{a_{i}}, p_{a_{i}}, c_{a_{i}}$ be the corresponding degrees and coefficients from their parameterizations (3.1) in the local chart $(z, w)=\left(\frac{1}{x}, \frac{y}{x}\right)$. Let

$$
c_{i}=\frac{c_{a_{i}}}{c_{b}}, W_{i}=Q_{p_{a_{i}}, q_{a_{i}}, c_{i}}(\theta)
$$

be the corresponding constants and polynomials from (3.15). Let $\theta_{i j}, i=$ $1, \ldots, l, j=1, \ldots, p_{a_{i}}$ denote the roots of the polynomials $W_{i}$.

Let $\Gamma_{b} \subset \Gamma$ denote the union of those germs in $\Gamma$ that are centered at $A$, tangent to $b$ and have Puiseux exponents greater than $r=r_{b}$ (some of them may be linear).

Let $k_{1}$ denote the number of those points $\xi(t)$ of the intersection $T_{t} b \cap \Gamma$, for which $x(\xi(t))=o(x(t))$, as $t \rightarrow A$. These are exactly those points that either lie in $\Gamma_{b}$ and have $x$-coordinates $o(x(t))$, see (3.13), or lie in those germs in $\Gamma$ that are not centered at $A$. Let $k_{2}$ denote the number of those points of the intersection $T_{t} b \cap \Gamma_{b}$ whose $x$-coordinates are asymptotic to $\frac{r}{r-1} x(t)$, see (3.12).

Proposition 3.13 Let $r=r_{b} \neq 2$. The collection of powers $\theta_{i j}^{q_{a_{i}}}$ of the above roots contains exactly $k_{1}$ powers equal to 2 and exactly $k_{2}$ powers equal to $\frac{r-2}{r-1}$. The collection $M$ of the other powers $\theta_{i j}^{q_{a_{i}}} \neq 2, \frac{r-2}{r-1}$ (each of the latter powers being taken with the total multiplicity of the corresponding roots) is invariant under the symmetry of the line $\mathbb{C}$ with respect to 1.

Proof The points of intersection $T_{t} b \cap \Gamma$ whose $x$-coordinates are $o(x(t))$ should be symmetric with respect to $t$ to other intersection points with $x$ coordinates asymptotically equivalent to $2 x(t)$ and vice versa. The latter should be points of intersection with the germs $a_{i}$. This follows from Proposition 3.12 and the fact that they cannot be points of the intersection $T_{t} b \cap \Gamma_{b}$. The latter statement follows from (3.12) and the inequality $\frac{r}{r-1} \neq 2$, which follows from the assumption that $r \neq 2$. This together with Corollary 3.11 and Proposition 3.12 implies that exactly $k_{1}$ powers $\theta_{i j}^{q_{a_{i}}}$ are equal to 2 . Similarly, if the collection $\Gamma_{b}$ is non-empty, then the intersection $T_{t} b \cap \Gamma_{b}$ contains points whose $x$-coordinates are asymptotic to $\frac{r}{r-1} x(t)$, by (3.12). Vice versa, the points of the intersection $T_{t} b \cap \Gamma$ with the latter asymptotics lie in $T_{t} b \cap \Gamma_{b}$. This follows from Corollary 3.11. Proposition 3.12 and the fact that they cannot be points of intersection with the germs $a_{i}$ : no number $s=\left(\frac{r}{r-1}\right)^{\frac{1}{q_{a_{i}}}}$ can be a root of a polynomial $W_{i}$ with $c_{i} \neq 0$. Indeed,

$$
W_{i}(s)=(r-1) s^{p_{a_{i}}}-r s^{p_{a_{i}}}\left(\frac{r}{r-1}\right)^{-1}+c_{i}=c_{i} \neq 0 .
$$


The above points of the intersection $T_{t} b \cap \Gamma_{b}$ should be symmetric to the points of the intersection $T_{t} b \cap \Gamma$ with $x$-coordinates asymptotically equivalent to $\left(2-\frac{r}{r-1}\right) x(t)=\frac{r-2}{r-1} x(t)$. The latter points of intersection lie in the union of the germs $a_{i}$, by Proposition 3.12 and due to $\frac{r-2}{r-1} \neq \frac{r}{r-1}$. Therefore, exactly $k_{2}$ powers $\theta_{i j}^{q_{a_{i}}}$ are equal to $\frac{r-2}{r-1}$. The points of the intersection $T_{t} b \cap \Gamma$ having $x$-coordinates asymptotically equivalent to $v x(t)$ with $v \neq 0,2, \frac{r}{r-1}, \frac{r-2}{r-1}$ are symmetric with respect to $t$, by relative symmetry property. The collection of the corresponding asymptotic factors $v$, thus symmetric with respect to 1 , coincides with the collection $M$ of the powers $\theta_{i j}^{q_{a_{i}}} \neq 2, \frac{r-2}{r-1}$, by the above arguments. The proposition is proved.

Proposition 3.14 Let $r>1$. Consider a collection

$$
S_{r}=\left\{\left(p_{i}, q_{i}, c_{i}\right)\right\}_{i=1, \ldots, N}, q_{i}, p_{i} \in \mathbb{N}, p_{i}>q_{i}, \frac{p_{i}}{q_{i}}=r, c_{i} \in \mathbb{C} \backslash\{0\},
$$

set $W_{i}(\theta)=(r-1) \theta^{p_{i}}-r \theta^{p_{i}-q_{i}}+c_{i}$. Let $\theta_{i j}\left(j=1, \ldots, p_{i}\right)$ denote the roots of the polynomials $W_{i}$. Let $M$ denote the collection of those $q_{i}$-th powers of roots $\theta_{i j}$ that are different from 2 and $\frac{r-2}{r-1}$ : each power being taken with the total multiplicity of the corresponding roots. Let $M$ be invariant under the symmetry of the line $\mathbb{C}$ with respect to 1 . Let $k_{1}\left(k_{2}\right)$ denote the number of those pairs $(i, j)$, for which $\theta_{i j}^{q_{i}}$ equals 2 (respectively, $\left.\frac{r-2}{r-1}\right)$. Set

$$
\Pi=\sum_{i} p_{i}=\text { the cardinality of the collection of all the roots } \theta_{i j} \text {. }
$$

Then

$$
(r-2) \Pi=k_{2}-k_{1}(r-1) .
$$

Proof The invariance of the collection $M$ under the symmetry with respect to 1 implies that the sum of its elements equals the cardinality $\operatorname{card}(M)=$ $\Pi-k_{1}-k_{2}$. On the other hand,

$$
\Pi-k_{1}-k_{2}=\sum_{x \in M} x=\sum_{i, j} \theta_{i j}^{q_{i}}-2 k_{1}-\frac{r-2}{r-1} k_{2},
$$

by definition. Let us calculate the latter right-hand side. One has

$$
\sum_{i j} \theta_{i j}^{q_{i}}=\sum_{i} \frac{p_{i}}{r-1}=\frac{\Pi}{r-1}
$$


as in (3.17). Substituting (3.21) to (3.20) yields

$$
\frac{\Pi}{r-1}-2 k_{1}-\frac{r-2}{r-1} k_{2}=\Pi-k_{1}-k_{2},
$$

which is equivalent to (3.19).

Proposition 3.15 Let $r>1$. Consider a collection $S_{r}$ as in (3.18), set $W_{i}(\theta)=(r-1) \theta^{p_{i}}-r \theta^{p_{i}-q_{i}}+c_{i}$. Let $\theta_{i j}\left(j=1, \ldots, p_{i}\right)$ denote the roots of the polynomials $W_{i}$, set $\Pi=\sum_{i=1}^{N} p_{i}$. Let $k_{1}\left(k_{2}\right)$ denote the number of index pairs $(i, j)$ for which $\theta_{i j}^{q_{i}}$ equals 2 (respectively, $\left.\frac{r-2}{r-1}\right)$. Let equality (3.19) hold. Then $r=2$.

Proof Let us represent $r$ as an irreducible fraction $r=\frac{p}{q}, p, q \in \mathbb{N}$. One has

$$
p_{i}=s_{i} p, q_{i}=s_{i} q, s_{i}=\operatorname{gcd}\left(p_{i}, q_{i}\right) .
$$

Suppose the contrary: equality (3.19) holds and $r \neq 2$.

Case 1): $r>2$. Hence, $r-2 \geq \frac{1}{q}$. One has

$$
k_{2} \geq(r-2) \Pi \geq \frac{1}{q} \Pi>\frac{1}{p} \Pi,
$$

by (3.19). This implies that there exists a polynomial $W_{i}$ for which more than $\frac{1}{p}$-th part of its roots have $q_{i}$-th powers equal to $\frac{r-2}{r-1}$. Thus, the number of the latter roots is no less than $s_{i}+1=\frac{p_{i}}{p}+1$. We will show that the above $W_{i}$ cannot exist. Let it exist, and let us fix it. None of its roots $\theta_{i j}$ with $\theta_{i j}^{q_{i}}=\frac{r-2}{r-1}$ can be a multiple root. Indeed, the derivative of the polynomial $W_{i}(\theta)$ equals

$$
\theta^{p_{i}-q_{i}-1}\left(p_{i}(r-1) \theta^{q_{i}}-r\left(p_{i}-q_{i}\right)\right)=p_{i}(r-1) \theta^{p_{i}-q_{i}-1}\left(\theta^{q_{i}}-1\right),
$$

since $r\left(p_{i}-q_{i}\right)=r q_{i}(r-1)=p_{i}(r-1)$. Therefore, the $q_{i}$-th powers of the roots of the derivative are equal to $0,1 \neq \frac{r-2}{r-1}$. Hence, $W_{i}$ has at least $s_{i}+1$ distinct roots $\theta=\theta_{i j}$ with $\theta_{i j}^{q_{i}}=\frac{r-2}{r-1}$. By definition, the latter roots satisfy the equality

$$
\begin{gathered}
\theta^{p_{i}-q_{i}}\left((r-1) \theta^{q_{i}}-r\right)+c_{i}=\theta^{p_{i}-q_{i}}\left((r-2-r)+c_{i}\right. \\
=-2 \theta^{p_{i}-q_{i}}+c_{i}=0
\end{gathered}
$$

and have equal $q_{i}$-th powers. Hence, their $p_{i}$-th powers are also equal, by (3.23). Therefore, the ratio of any two above roots is simultaneously a $q_{i}$-th 
and $p_{i}$-th root of unity, and hence, an $s_{i}$-th root of unity, since $p_{i}=s_{i} p$, $q_{i}=s_{i} q$ and $p, q$ are coprime. Therefore, the number of roots under question is no greater than $s_{i}$. We get a contradiction.

Case 2): $1<r<2$. One has

$$
k_{1} \geq \Pi \frac{2-r}{r-1}=\Pi \frac{2 q-p}{p-q} \geq \frac{1}{p-q} \Pi>\frac{1}{p} \Pi,
$$

by (3.19). This implies that the there exists a polynomial $W_{i}$ that has at least $s_{i}+1$ roots whose $q_{i}$-th powers are equal to 2 , as in the previous case. We then get a contradiction, as in the above discussion. The proposition is proved.

Lemma 3.16 Let $r>1, S_{r}=\left\{\left(p_{i}, q_{i}, c_{i}\right)\right\}_{i=1, \ldots, N}$ be a collection as in (3.18). Set $W_{i}(\theta)=(r-1) \theta^{p_{i}}-r \theta^{p_{i}-q_{i}}+c_{i}$. Let $\theta_{i j}\left(j=1, \ldots, p_{i}\right)$ denote the roots of the polynomials $W_{i}$. Let $M$ denote the collection of those $q_{i}$-th powers of roots $\theta_{i j}$ that are different from 2 and $\frac{r-2}{r-1}$ : each power being taken with the total multiplicity of the corresponding roots. Let $M$ be invariant under the symmetry of the line $\mathbb{C}$ with respect to 1 . Then $r=2$.

Lemma 3.16 follows from Propositions 3.14 and 3.15 . Lemma 3.16 together with Proposition 3.13 imply the statement of Theorem 3.6 .

\section{Invariants of singularities and Plücker formulas. Proofs of Theorems 1.6 and 1.5}

Here we prove Theorem 1.6 in Subsection 4.2. Then we prove Theorem 1.5 in Subsection 4.3. Afterwards in Subsection 4.4 we state and prove a purely algebraic-geometric Theorem 4.1 that is a direct consequence of Theorems 3.6 and 1.6 that seems to be of independent interest.

The proof of Theorem [1.6 essentially uses general Plücker and genus formulas for plane algebraic curves; the corresponding background material is presented in Subsection 4.1. The main observation is that the upper bound 2 to the projective Puiseux exponents of all transverse local branches of the curve and Plücker formulas yield that the singularity invariants of the considered curve must obey a relatively high lower bound. On the other

hand, the contribution of the points in the infinite line $\overline{\mathbb{C}}_{\infty}$ appears to be not sufficient to fit that lower bound unless the curve is a conic. 


\subsection{Invariants of plane curve singularities}

For the reader's convenience, we recall here main definitions and formulas. Almost all this stuff is classically known (see [12, Chapter III], [20, §10], and the modern exposition in [16, Section I.3]).

Let $\left(x_{0}: x_{1}: x_{2}\right)$ be homogeneous coordinates on $\mathbb{C P}^{2}$. Let $\alpha \subset \mathbb{C P}^{2}$ be a reduced, irreducible curve of degree $d>1$, i.e., given by a homogeneous square-free, irreducible polynomial $F\left(x_{0}, x_{1}, x_{2}\right)$ of degree $d>1$. For any point $A \in \alpha$ and a sufficiently small closed ball $V \subset \mathbb{C P}^{2}$ centered at $A$ the intersection $\alpha \cap V$ is topologically a bouquet of discs $b_{i}, 1 \leq i \leq r$, given by the local branches of the germ $(\alpha, A)$.

(1) Multiplicity and dual multiplicity of a local branch. Given a local branch $b_{i}$ of the curve $\alpha$ at $A \in \alpha$ and its Puiseux parametrization (3.1), the number $s\left(b_{i}\right)=q$ is called the multiplicity, and the number $s^{*}\left(b_{i}\right)=p-q$ the dual multiplicity of the branch $b_{i}$. Note that $s\left(b_{i}\right)$ is the intersection multiplicity of the branch $b_{i}$ with a transversal line, while $s^{*}\left(b_{i}\right)+s\left(b_{i}\right)$ is the intersection multiplicity with the tangent line $T_{A} b_{i}$. Observe also that the subquadraticity condition for $b_{i}$ is equivalent to the relation

$$
s^{*}\left(b_{i}\right) \leq s\left(b_{i}\right)
$$

(2) $\delta$-invariant. Let $f(x, y)=0$ be an equation of the germ $(\alpha, A)$ (just $F=0$ rewritten in local coordinates $x, y)$. Then $\alpha_{\varepsilon}:=\{f(x, y)=\varepsilon\} \cap V$, for $0<|\varepsilon| \ll 1$, is a smooth surface with $r$ holes (Milnor fiber). The $\delta$-invariant of the germ $(\alpha, A)$ admits several equivalent definitions and topologically can be defined as the genus of the closed surface obtained by attaching a sphere with $r$ holes to the surface $\alpha_{\varepsilon}$. The genus formula in the form of Hironaka 17, reads

$$
\frac{(d-1)(d-2)}{2}=g(\alpha)+\sum_{A \in \operatorname{Sing}(\alpha)} \delta(\alpha, A),
$$

where $g(\alpha)$ is the geometric genus of $\alpha$, i.e., the genus of the Riemann surface obtained by the resolution of singularities of $\alpha$. In particular, we have

$$
\sum_{A \in \operatorname{Sing}(\alpha)} \delta(\alpha, A) \leq \frac{(d-1)(d-2)}{2} .
$$

(3) Class of the singular point ( $\kappa$-invariant). Given a germ $(\alpha, A)$ and local affine coordinates $(x, y)$, suppose that the $y$-axis is not tangent to any 
of the local branches $b_{i}, 1 \leq i \leq r$. Denote by $\alpha^{\prime}$ the polar curve of $\alpha$ defined by the equation $\frac{\partial f}{\partial y}=0$. The class of the germ $(\alpha, A)$ is defined by

$$
\kappa(\alpha, A)=\left(\alpha \cdot \alpha^{\prime}\right)_{A},
$$

the intersection multiplicity of $\alpha$ and $\alpha^{\prime}$ at $A$. It is well-known (see, for instance, [16, Propositions I.3.35 and I.3.38]) that

$$
\kappa(\alpha, A)=2 \delta(\alpha, A)+\sum_{i=1}^{r}\left(s\left(b_{i}\right)-1\right) .
$$

(4) Hessian of the singular (or inflection) point. The Hessian $H_{\alpha}$ of the curve $\alpha$ is the curve given by the equation $\operatorname{det}\left(\frac{\partial^{2} F}{\partial x_{i} \partial x_{j}}\right)_{0 \leq i, j \leq 2}=0$. The Hessian of the germ $(\alpha, A)$ is

$$
h(\alpha, A)=\left(\alpha \cdot H_{\alpha}\right)_{A},
$$

the intersection multiplicity of $\alpha$ and $H_{\alpha}$ at $A$. It vanishes in all smooth points of $\alpha$, where $\alpha$ quadratically intersects its tangent line. An expression for $h(\alpha, A)$ via the preceding invariants was found in [23, Formula (2)]. It can be written as

$$
h(\alpha, A)=3 \kappa(\alpha, A)+\sum_{i=1}^{r}\left(s^{*}\left(b_{i}\right)-s\left(b_{i}\right)\right) .
$$

In view of $\operatorname{deg} H_{\alpha}=3(d-2)$, Bézout's theorem yields (a Plücker formula)

$$
3 d(d-2)=\sum_{A \in \alpha} h(\alpha, A) .
$$

\subsection{Proof of Theorem 1.6}

Let $\alpha \subset \mathbb{C P}^{2}$ be a curve of degree $d \geq 2$, satisfying the hypotheses of Theorem 1.6. We will show that $d=2$.

Observe that $\delta(\alpha, A)=\kappa(\alpha, A)=h(\alpha, A)=0$ for all points $A \in \alpha \backslash \overline{\mathbb{C}}_{\infty}$. Denote by $\mathcal{B}_{\text {tr }}$, resp. $\mathcal{B}_{\text {tan }}$ the set of the local branches of $\alpha$ centered on $\overline{\mathbb{C}}_{\infty}$ and transversal, resp. tangent to $\overline{\mathbb{C}}_{\infty}$. Relation (4.1) holds for all the local branches $b \in \mathcal{B}_{t r}$, since they are subquadratic, by the condition of Theorem 1.6. Thus, their contributions to the sum $\sum_{i}\left(s^{*}\left(b_{i}\right)-s\left(b_{i}\right)\right)$ are non-positive. Therefore, from (4.4) and (4.5), we get

$$
3 d(d-2) \leq 3 \sum_{A \in \alpha \cap \overline{\mathbb{C}}_{\infty}} \kappa(\alpha, A)+\sum_{b \in \mathcal{B}_{\text {tan }}}\left(s^{*}(b)-s(b)\right) .
$$


Together with (4.2) and (4.3) this yields

$$
\begin{gathered}
3 d(d-2) \leq 6 \sum_{A \in \alpha \cap \overline{\mathbb{C}}_{\infty}} \delta(\alpha, A)+3 \sum_{b \in \mathcal{B}_{t r} \cup \mathcal{B}_{\text {tan }}}(s(b)-1)+\sum_{b \in \mathcal{B}_{\text {tan }}}\left(s^{*}(b)-s(b)\right) \\
\leq 3(d-1)(d-2)+3 \sum_{b \in \mathcal{B}_{\text {tr }} \cup \mathcal{B}_{\text {tan }}}(s(b)-1)+\sum_{b \in \mathcal{B}_{\text {tan }}}\left(s^{*}(b)-s(b)\right) \\
=3(d-1)(d-2)+\sum_{b \in \mathcal{B}_{t r} \cup \mathcal{B}_{\text {tan }}}(s(b)-1)+\sum_{b \in \mathcal{B}_{t r}} s(b) \\
+\sum_{b \in \mathcal{B}_{t r}}(s(b)-2)+\sum_{b \in \mathcal{B}_{t a n}}\left(s^{*}(b)+s(b)-2\right) .
\end{gathered}
$$

Developing $d=\left(\alpha \cdot \overline{\mathbb{C}}_{\infty}\right)$ into contributions of local branches $b \in \mathcal{B}_{t r} \cup \mathcal{B}_{\text {tan }}$, we obtain

$$
\left\{\begin{array}{c}
\sum_{b \in \mathcal{B}_{t r} \cup \mathcal{B}_{\text {tan }}}(s(b)-1)=d-\left|\mathcal{B}_{\text {tr }} \cup \mathcal{B}_{\text {tan }}\right|-\sum_{b \in \mathcal{B}_{\text {tan }}} s^{*}(b) \\
\leq d-\left|\mathcal{B}_{\text {tr }}\right|-2\left|\mathcal{B}_{\text {tan }}\right| \\
\sum_{b \in \mathcal{B}_{t r}} s(b)=d-\sum_{b \in \mathcal{B}_{\text {tan }}}\left(s^{*}(b)+s(b)\right) \leq d-2\left|\mathcal{B}_{\text {tan }}\right| \\
\sum_{b \in \mathcal{B}_{t r}}(s(b)-2)+\sum_{b \in \mathcal{B}_{\text {tan }}}\left(s^{*}(b)+s(b)-2\right) \\
=d-2\left|\mathcal{B}_{\text {tr }} \cup \mathcal{B}_{\text {tan }}\right|
\end{array}\right.
$$

and hence the sequence of relations (4.7) reduces to

$$
2 \geq\left|\mathcal{B}_{\text {tr }}\right|+2\left|\mathcal{B}_{\text {tan }}\right|
$$

If $\mathcal{B}_{\text {tan }}=\emptyset$ and all the branches $b \in \mathcal{B}_{\text {tr }}$ are centered at one point, then $\sum_{b \in \mathcal{B}_{t r}} s(b)=d$, and the intersection multiplicity of $\alpha$ with the tangent to one of the branches $b \in \mathcal{B}_{t r}$ appears to be greater than $d$. This implies that the latter tangent line is contained in $\alpha$. Thus, $\alpha$ splits off a line, contrary to the irreducibility assumption.

If $\mathcal{B}_{\text {tan }}=\emptyset,\left|\mathcal{B}_{t r}\right|=2$, and the two branches $b_{1}, b_{2} \in \mathcal{B}_{t r}$ have distinct centers, we have an equality in (4.9); hence, equalities in all the above relations, in particular, in (4.6). Thus, in view of (4.4), (4.5) and the inequality $s^{*}\left(b_{i}\right) \leq s\left(b_{i}\right)$ (subquadraticity), it means $s^{*}\left(b_{i}\right)=s\left(b_{i}\right), i=1,2$. Intersecting $\alpha$ with the tangent lines to $b_{1}$ and $b_{2}$, we obtain $s\left(b_{i}\right) \leq \frac{d}{2}, i=1,2$, while the intersection with $\overline{\mathbb{C}}_{\infty}$ yields $s\left(b_{1}\right)+s\left(b_{2}\right)=d$. It follows that $s\left(b_{1}\right)=s\left(b_{2}\right)=\frac{d}{2}$. Choosing affine coordinates in $\mathbb{C P}^{2} \backslash \overline{\mathbb{C}}_{\infty}$ so that the coordinate axes are tangent to $b_{1}$ and $b_{2}$ (at infinity) respectively, we obtain that the Newton polygon of the defining polynomial of $\alpha$ is just the segment 
$[(0,0),(d / 2, d / 2)]$. Indeed, in local affine coordinates $z_{1}, w_{1}$ in a neighborhood of the center $A$ of the branch $b_{1}$ such that the tangent line $T_{A} b_{1}$ is the $z_{1}$-axis and $\overline{\mathbb{C}}_{\infty}$ is the $w_{1}$-axis, we have $b_{1}$ given by

$$
z_{1}=t^{d / 2}, \quad w_{1}=c_{1} t^{d}(1+o(1)), \quad t \in(\mathbb{C}, 0)
$$

which means that the Newton diagram of $\alpha$ in these coordinates is the segment $[(d, 0),(0, d / 2)]$, i.e., the coefficients of all the monomials $z_{1}^{i} w_{1}^{j}$ with $(i, j)$ below this segment vanish. In the coordinates $x=\frac{1}{z_{1}}, y=\frac{w_{1}}{z_{1}}$, this yields that the coefficients of all monomials $x^{i} y^{j}$ with $j<i$ vanish. The same consideration with the affine coordinates $z_{2}, w_{2}$ in a neighborhood of the center $B$ of the branch $b_{2}$ such that $T_{B} b_{2}$ is the $z_{2}$-axis and $\overline{\mathbb{C}}_{\infty}$ is the $w_{2^{-}}$ axis leads to the conclusion that the coefficients of all monomials $x^{i} y^{j}$ with $j>i$ vanish. This finally leaves the only Newton segment $[(0,0),(d / 2, d / 2)]$. Note that a polynomial with such a Newton segment factors into the product of $\frac{d}{2}$ binomials of type $x y-\lambda$. Thus, $d=2$ due to the irreducibility of $\alpha$.

If $\left|\mathcal{B}_{\text {tan }}\right|=1$ and $\left|\mathcal{B}_{t r}\right|=0$, then we have an equality in (4.9); hence, all the above inequalities turn to be equalities, in particular, the second relation in (4.8), that is, $s^{*}(b)+s(b)=2$ for the unique branch of $\alpha$ centered on $\overline{\mathbb{C}}_{\infty}$, which finally means that $d=2$.

\subsection{Proof of Theorem 1.5}

Let $\gamma$ denote the complex projective Zariski closure of the curve $C$. Let $\alpha$ be its arbitrary nonlinear irreducible component. The curve $\alpha$ has neither singular, nor inflection points in $\mathbb{C}^{2}$ (Theorem 2.2), and each its local branch transverse to the infinity line (if any) is quadratic, by Corollary 3.7. Therefore, $\alpha$ is a conic, by Theorem 1.6. Thus, the curve $\gamma$ is a finite union of conics and lines, and $C$ is a union of arcs of conics and lines. The latter union of arcs is finite: each potential end of an arc should be a singular point of the curve $\gamma$, and the number of singular points of an algebraic curve is finite. At least one conical arc is present, since $C$ is a $C^{4}$-smoothly immersed curve that does not lie in a line. The parameter interval (circle) of the curve $C$ is thus split into a finite number of segments, each of them parameterizes an entire arc of conic (line) in $C$. Any two arcs parameterized by adjacent segments have contact of order at least 5 , since $C$ is a $C^{4}$-smoothly immersed curve. Therefore, no conical arc can be adjacent to a linear arc, which implies that there are no linear arcs at all. No two arcs of distinct conics can be adjacent neither: otherwise, their intersection index would be greater than 4 , by the above statement. This implies that $C$ lies in just one conic and proves Theorem 1.5 . 


\subsection{Appendix: a general corollary of Theorems 3.6 and 1.6}

The following theorem is a direct consequence of Theorems [3.6 and 1.6.

Theorem 4.1 Let $\alpha \subset \mathbb{C P}^{2}$ be an irreducible algebraic curve distinct from a line such that all its singular and inflection points (if any) lie in the infinity line $\overline{\mathbb{C}}_{\infty}$. Let for every point $A \in \alpha \cap \overline{\mathbb{C}}_{\infty}$ each local branch $\beta$ of the curve $\alpha$ at $A$ that is transverse to the infinity line $\overline{\mathbb{C}}_{\infty}$ (if any) have the local relative symmetry property with respect to some $\left(T_{A} \beta, A\right)$-local multigerm $\Gamma=\Gamma(\beta)$. Then $\alpha$ is a conic.

Proof Each local branch $\beta$ as above is quadratic (Theorem 3.6). Hence, $\alpha$ is a conic, by Theorem 1.6.

\section{Acknowledgements}

We are grateful to Misha Bialy, Andrey Mironov and Sergei Tabachnikov for introducing us to polynomially integrable billiards, helpful discussions and providing the starting point for our work: Theorem 2.2. This work was partly done during the visits of the first author (A.Glutsyuk) to Sobolev Institute at Novosibirsk and to Tel Aviv University. He wishes to thank Andrey Mironov and Misha Bialy for their invitations and hospitality and both institutions for their hospitality and support. We are grateful to the referee for a very careful reading of the paper and very helpful remarks, which also led to major improvement of the first author's paper [14].

\section{References}

[1] Amiran, E. Caustics and evolutes for convex planar domains. J. Diff. Geometry, 28 (1988), 345-357.

[2] Avila, A.; De Simoi, J.; Kaloshin, V. An integrable deformation of an ellipse of small eccentricity is an ellipse. Ann. of Math. (2) 184 (2016), no. $2,527-558$.

[3] Bialy, M. Convex billiards and a theorem by E. Hopf. Math. Z., 214(1) (1993), 147-154.

[4] Bialy, M. Hopf rigidity for convex billiards on the hemisphere and hyperbolic plane. Discrete Contin. Dyn. Syst. 33 (2013), no. 9, 3903-3913. 
[5] Bialy, M. On totally integrable magnetic billiards on constant curvature surface. Electron. Res. Announc. Math. Sci. 19 (2012), 112-119.

[6] Bialy, M.; Mironov, A. A. Angular billiard and Angular Birkhoff Conjecture. Adv. in Math. 313 (2017), 102-126.

[7] Bialy, M.; Mironov, A. On fourth-degree polynomial integrals of the Birkhoff billiard. Proc. of Steklov Inst. of Math., 295 (2016), no. 1, 27-32.

[8] Bialy, M.; Mironov, A. Algebraic Birkhoff conjecture for billiards on Sphere and Hyperbolic plane. J. Geom. Phys., 115 (2017), 150-156.

[9] Boyland, Ph. Dual billiards, twist maps and impact oscillators. Nonlinearity 9 (1996), 1411-1438.

[10] Bolotin, S.V. Integrable Birkhoff billiards. Mosc. Univ. Mech. Bull. 45:2 (1990), 10-13.

[11] Bolotin, S.V. Integrable billiards on surfaces of constant curvature. Math. Notes 51 (1992), No. 1-2, 117-123.

[12] Brieskorn, E., and Knörrer, H. Plane algebraic curves. Birkhäuser, Basel, 1986.

[13] Glutsyuk, A. On quadrilateral orbits in complex algebraic planar billiards. Moscow Math. J., 14 (2014), No. 2, 239-289.

[14] Glutsyuk, A. On polynomially integrable Birkhoff billiards on surfaces of constant curvature. Preprint https://arxiv.org/abs/1706.04030

[15] Glutsyuk, A. On two-dimensional polynomially integrable Birkhoff billiards on surfaces of constant curvature. To appear in Doklady Mathematics.

[16] Greuel, G.-M., Lossen, C., and Shustin, E. Introduction to singularities and deformations. Springer, Berlin, 2007.

[17] Hironaka, H. Arithmetic genera and effective genera of algebraic curves. Mem. Coll. Sci. Univ. Kyoto. Sect. A30 (1956), 177-195.

[18] Kaloshin, V.; Sorrentino, A. On local Birkhoff Conjecture for convex billiards. To appear in Annals of Math. Also available as preprint https://arxiv.org/abs/1612.09194 
[19] Kozlov, V.V.; Treshchev, D.V. Billiards. A genetic introduction to the dynamics of systems with impacts. Translated from Russian by J.R.Schulenberger. Translations of Mathematical Monographs, 89, Americal Mathematical Society, Providence, RI, 1991.

[20] Milnor, J. Singular points of complex hypersurfaces. Princeton Univ. Press, Princeton, 1968.

[21] Moser, J. Is the solar system stable? Math. Intell. 1 (1978), 65-71.

[22] Poritsky, H. The billiard ball problem on a table with a convex boundary - an illustrative dynamical problem. Ann. of Math. (2), 51 (1950), 446470 .

[23] Shustin, E. On invariants of singular points of algebraic curves. Math. Notes of Acad. Sci. USSR 34 (1983), 962-963.

[24] Tabachnikov, S. Billiards. Panor. Synth. 1 (1995), vi+142.

[25] Tabachnikov, S. Geometry and billiards. Student Mathematical Library 30, American Mathematical Society, Providence, RI, 2005.

[26] Tabachnikov, S.; Dogru, F. Dual billiards. Math. Intelligencer 27:4 (2005), 18-25.

[27] Tabachnikov, S. On algebraically integrable outer billiards. Pacific J. of Math. 235 (2008), no. 1, 101-104.

[28] Treschev, D. Billiard map and rigid rotation. Phys. D., 255 (2013), $31-34$.

[29] Treschev, D. On a Conjugacy Problem in Billiard Dynamics. Proc. Steklov Inst. Math., 289 (2015), No. 1, 291-299.

[30] Treschev, D. A locally integrable multi-dimensional billiard system. Discrete Contin. Dyn. Syst. 37 (2017), no. 10, 5271-5284.

[31] Wojtkowski, M.P. Two applications of Jacobi fields to the billiard ball problem. J. Differential Geom. 40 (1) (1994), 155-164. 